\title{
LA CONVENTION DE LONDRES SUR LE STATUT DES FORCES DE L'OTAN ET LE PRINCIPE DE TERRITORIALITE EN DROIT PENAL INTERNATIONAL
}

\author{
Dr. Dưrmuş TEZCAN*
}

\section{Introduction}

1. Le principe de territorialité est le principe en vertu duquel "tout ce qui se produit sur un territoire déterminé relève de la loi en vigueur sur ce territoire". ${ }^{1}$

Suivant la définition donnée par M. Donnedieu de Vabres, sous l'angle du droit pénal international, "il a pour objet l'affirmation de' la compétence exclusive d'un Etat, de ses juridictions et de ses lois propres, à l'égard de tous les actes punissables qui ont été commis sur son territoire". ${ }^{2}$

Le principe de territorialité a en soi, et du point de vue théorique, une portée exclusive sur les plans législatif et juridictionnel: il signifie que l'infraction est nécessairement régie par la loi en vigueur sur le territoire où elle a été commise, et que les juridictions dé cet Etat sont seules compétentess pour en connaitre. ${ }^{3}$

Bien entendu, cette portée ne lui est pas reconnue de manière exclusive en droit pénal international positif, puisque les Etats ont en fait d'autres chefs de compétence (compétences personnelle, réelle et universelle) et qu'ils

* Chargé de cours associé à la Fac. de Sc. pol. d'Ankara

1 Niboyet, "L'Universalité des règles de solution des conflits" in Revue Critique de Droit international privé, 1950, pp. 509 et ss. (v.p. 513).

$2 \mathrm{H}$. Donnedieu de Vabres, Indroduction à l'étude du droit pénal international, Paris, Librairie Recueil-Sirey, 1922, p. 11.

3 D. Tezcan, Territorialité et conflits de juridictions en droit pénal international, Thèse de doctorat, Univ, Libre de Bruxelles, 1976, pp. 86 et ss 
n'admettent que dans une mesure limitée l'application d'un droit pénal étranger.

Par ailleurs, on constate que l'évolution du droit pénal international va dans le sens d'un amenuisement du critère strictement territorial de ce droit.

Dans cette optique, il est intéressant d'examiner la coopération atlantique instaurée par la Convention de Londres sur le statut des forces de l'OTAN, à laquelle la Turquie est partie.

2. L'Organisation du Traité de l'Atlantique Nord est issue du Traité signé à Washington le 4 avril 1949 entre la Belgique, le Canada, le Danemark, les Etats-Unis, la France, l'Islande, l'Italie, le Luxembourg, la Norvège, les Pays-Bas, le Portugal et le Royaume-Uni. Elle groupe actuellement seize Etats, la Grèce et la Turquie ayant accédé à l'Organisation le 18 février 1951, l'Allemagne le 5 mai 1955 et l'Espagne le 29 mai 1982.

Nous nous proposons d'examiner ici uniquement le système instauré par l'OTAN au regard des conflits de juridictions qui sont suscités par la présence de troupes à l'étranger. Car "le séjour des Forces armées d'un Etat sur le territoire d'un autre intéresse la souveraineté de l'Etat dont relèvent ces forces armées et la souveraineté de celui où stationnent ces troupes. C'est de commun accord que les Etats établissent des règles en vue d'éviter lis conflits que la confrontation de leurs souverainetés et de leurs intérêts ne manquerait pas de créer".4

L'OTAN n'est certes pas la seule organisation à laquelle ce problème ait été posé. ${ }^{5}$ Mais c'est sans doute elle qui,

${ }^{4}$ M. Danse, "Le statut pénal de l'OTAN", Rev. de droit mil. et de droit de la guerre, 1963-64, pp. 77 et ss.; v. ég. O. Debbasch, "L'occupation militaire", Paris, Libr. gén. de droit et de jurisprudence, 1962; S. Lazareff, "Le statut de l'OTAN et son application en France", Paris, Ed. A. Pedone, 1964, pp. 2 et ss.; J. Verhoeven, "Le statut des bases militaires étrangères et des quartiers généraux interalliés implantés en Belgique", Rev. Belge de Dr. int. 1969, pp. 570 et ss.

5 En dehors de l'échec de la création d'une Communauté européenne de défense (và ce sujet R. Aron et D. Lerner, "La querelle de la C.E.D.", Paris, Libr. A. Colin; J. Gilissen, "L'application c'es lois pénales 
la première, a tenté d'apporter une solution aux conflits de' juridictions en matière pénale.

La coopération atlantique en ce domaine résulte de l'article VII de la Convention entre les Etats parties au Traité de l'Atlantique Nord sur le statut de leurs forces, signée à Londres le 19 juin 1951, que nous appellerons par simplification la Convention de Londres sur le statut des forces de l'OTAN.

L'article VII établit une distinction de base entre, d'une part, les cas de compétence exclusive' de l'Etat d'origine cu de l'Etat de séjour (chiffres 1 et 2) et, d'autre part, les cas de compétence concurrente des deux Etats (chiffres 3 et suivants).

La Convention de Londres sur le statut des forces de l'OTAN a fait l'objet de très nombreuses études, auxquelles le lecteur pourra utilement se référer. Notre examen sera centré sur les rapports entre les compétences de l'Etat d'origine et de l'Etat de séjour car c'est le point qui présente le plus d'intérêt au regard de l'étude du rôle du principe de territorialité.

aux militaires à l'étranger dans les rapports intraeuropéens" in "Droit pénal européen", op. cit., pp. 299 et ss.; Claude Delmas, "Histoire des projets d'unification politique de l'Europe", BruxellesNamur, Ed. U.G.A. - Heule, 1971, pp. 104 et ss.; C. Melchior de Molenès, "Réflexions sur la défense européenne" in Les Problèmes de l'Europe, $1971, \mathrm{n}^{\circ}$ 54, pp. 31-32 et 83-85; W. Schutze, "Les problèmes de la défense européenne" in Les problèmes de l'Europe, 1972, $\mathrm{n}^{\circ} 55, \mathrm{pp}$. 15 et. ss.), on doit faire mention du Traité d'amitié, de coopération et d'assistance mutuelle signé à Varsovie le 14 mai 1955 entre I'U.R.S.S. et les Etats du bloc oriental, qui contient des règles de conflits (v. à ce sujet Claude-A. Colliard, "Institutions internationales", op. cit., pp. 466 et ss.; M. Lachs, "Le Traité de Varsovie du 14 mai 1955", Ann. fr. dr. int. 1955, pp. 120 et ss.; John Gilissen, "L'application des lois pénales...", op. cit., pp. 314 et ss.). Il convient également de signaler que les Etats-Unis ont conclu plusieurs conventions bilatérales avec des Elats où leurs troupes stationnent (il y a plus de 700.000 soldats américains stationnés dans près de 70 pays) - v. à ce sujet J.B. Whitten, "L'exercice de la compétence pénale à l'égard des forces américaines à l'étranger", RGDIP 1959, pp. 5 et ss. 
II. La Convention de Londres sur le statut des forces de l'OTAN et le principe de territorialité

\section{A. Les cas donnant lieu à une compétence exclusive}

L'article' VII, chiffre 2 de la Convention dispose que :

“a. Les autorités militaires de l'Etat d'origine ont le droit d'exercer une juridiction exclusive sur les personnes soumises aux lois militaires de cet Etat, en ce qui concerne les infractions punies par la législation de l'Etat d'origine. notamment les infractions portant atteinte à la sûreté de cet Etat mais ne tombant pas sous le coup de la légis]ation de' l'Etat de séjour;

b. Les autorités de l'Etat de séjour ont le droit d'exercer une juridiction exclusive sur les membres d'une force ou d'un élément civil et sur les personnes à charge' en ce qui concerne les infractions punies par les lois de l'Etat de séjour, notamment les infractions portant atteinte à la sûreté de cet Etat mais ne tombant pas sous le coup de la législation de l'Etat d'origine;

c. Au sens du présent paragraphe (...), sont considérées comme infractions portant atteinte à la sûreté d'un Etat:

(i) la trahison,

(ii) le sabotage, l'espionnage ou la violation de la législation relative' aux secrets d'Etats ou de défense nationale".

A côté des infractions expressément citées dans le texte, on peut ranger parmi celles qui donnent lieu à compétence exclusive, d'une' part les délits spécifiquement militaires, tels que désertion, insubordination ou abandon de poste, et d'autre part les infractions strictement territoriales, c'est-à-dire celless qui, tombant uniquement sous le coup des lois pénales d'un pays déterminé, diffèrent, en raison de leur nature, d'une manière fondamentale d'un pays à l'autre, comme par exemplé les règles relatives à la priorité 
en matière de circulation routière. ${ }^{6}$ Toutefois, la plupart des Etats ont inséré dans leur droit national des dispositions en vertu desquelles certaines catégories d'infractions qui présentent en général ce caractère strictement territorial sont punissables dans l'Etat d'origine. C'est le cas de la Belgique (art. 57bis du Code pénal militaire), des PaysBas (art. 168 du Code pénal militaire), du Canada (art. 119 A de la Loi sur la défense nationale), des Etats-Unis (art. 134 de l'Uniform Code of military justice), de la GrandeBretagne (art. 69 de l'Army Act du 16 mai 1955) et de Ja France (art. 66 du Code de justice militaire du 16 mai 1965) .

En Belgique, la disposition spécifique a été insérée par une loi du 27 février 1958, et modifiée par une loi du 5 avril 1963. Nous en donnons ci-dessous le présent alinéa, à titre d'illustration de $c \in$ type de législation, qui constitue une remarquable application de la règle de la lex loci delicti commissi :

"Le militaire qui, sur le territoire d'un Etat étranger où il est en service, contrevient à la législation de cet Etat en matilère forestière, rurale, de chasse, de pêche, de circulation routière, de douanes, de change ou dé réglementation des importations ou exportations, serra puni d'un emprisonnement de huit jours à un an et d'une amende de 26 à $1.000 \mathrm{Fr}$. ou d'une de ces peines seulement.

Il en sera de même de la personne attachée à une fraction de l'armée se trouvant sur le territoire d'un Etat étranger ou autorisée à suivre un corps de troupe qui fait partie de cette fraction de l'armée, lorsque le fait est commis sur ce territoire".

Entre Etats qui ne disposent pas d'un texte exprès, la

6 v. à ce sujet John Gilissen, "L'application des lois pénales...", op. cit., p. 313 et p. 348.

7 v. à ce suję John Gilissen, "L'application des lois pénales...", op. cit., pp. 317 et ss.; Serge Lazareff, "Le statut des forces de l'OTAN et son application en France", op. cit., pp. 176 et ss.; sur les difficultès d'application de la loi étrangère, v. M. Lesieur, "L'application de la loi étrangère" Rev. de dr. pén. mil. 1970, pp. 77 à 90 et plus particulièrement chapitre III, pp. 86 à 88 . 
compétence' exlusive des juridictions de l'Etat de séjour est confirmée par la jurisprudence. ${ }^{8}$

\section{B. Les cas donnant lieu à compétence concurrente}

L'article VII, chiffre 3 établit à cet égard un système de juridiction prioritaire, avec possibilité de renonciation au profit de l'autre Etat. Nous envisagerons successivem nnt les hypothèses dans lesquelles l'Etat d'origine et l'Etat de séjour disposent de cette compétence prioritaire, pour examiner ensuite le système de renonciation.

\section{a) Compétence prioritaire de l'Etat d'origine}

Elle existe pour deux types d'infractions:

$1^{\circ}$ "Les infractions portant atteinte uniquement ̀े la sûreté ou à la propriété de cet Etat ou les infractions portant atteinte uniquement à la personne ou à la propriété d'un membre de la force, ou d'un élément civil de cet Etat ainsi que d'une personne à charge"' (chiffre 3. a. (i) ) .

Parmi les infractions portant atteinte à la sûreté de l'Etat d'origine, on doit ranger celle's qui portent principalement atteinte à la sûreté de cet Etat, mais qui sont considérées occasionnellement par l'Etat de séjour comme portant aussi atteinte à sa sûreté, par le fait que cé dernier Etat possède une législation spécifique en la matière. ${ }^{9}$

En ce qui concerne les infractions relatives à la propriété, c'est évidemment le vol qui en est la principale application pratique..$^{10}$

\footnotetext{
8 V. par exemple l'arrêt de la $2 e \mathrm{ch}$. crim. de la Cour de cassation turque du 20 juin 1055 qui admet cette règle en matière cle conduite automobile mettant en danger les personnes et les biens (cité par M. Kıran et C. Güneri, "NATO" (en turc), Ankara, Ed. Alnnyıldız, 1962, pp. 52-53) - v. cependant infra la jurisprudence turque récente lorsqu'il s'agit d'une infraction commise dans l'exécution du service.

9 Ainsi que le note S. Lazareff (op. cit., p. 185), "une telle solution aurait l'avantage de reconnaitre sur le plan de la sécurité la solidarité des nations atlantiques".

10 v. ¿̀ ce sujet Frédéric Stroh, "Le militaire et le vol", Rev. sc. cr. 1972 pp. 656-663.
} 
Le vol d'armes constitue à cet égard un problème délicat, car lorsque les armes sont introduites dans l'Etat de séjour, celui-ci peut invoquer une infraction distincte, le trafic illégal d'armes. Malgré les critiques qui sont formulées à ce sujet, la pratique des Etats permet en ce cas des poursuites séparées. ${ }^{11}$

La nationalité de la victime ne joue qu'un rôle limité. Le projet initial faisait de la nationalité de l'Etat d'origine de la personne à charge une condition de la compétence prioritaire, mais cette condition fut écartée dans le texte définitif..$^{12}$ Nous pensons que cette suppression est regrettable. En effet, lorsque la personne à charge garde sa nationalité d'origine, c'est en général qu'elle ne désire pas s'intégrer définitivement à l'Etat d'origine, et le fait que la poursuite y soit exercée, devant des juridictions militaires où la constitution de partie civile n'est en général pas admise, peut lui porter préjudice.

Lorsque la victime n'est ni un membre de la force, ni une personne à charge, l'Etat d'origine ne peut bénéficier de la priorité que si elle en possède la nationalité. ${ }^{13}$

\section{$2^{\circ}$ "Les infractions résultant de tout acte ou négli-}

11 v. à ce sujet S. Lazareff, op. cit., p. 186; J.M. Snee et K.A. Pye, "Statuts of forces agreement - Criminal jurisdiction", New-York, Oceana publications, pp. 55 et ss.; Nous estimons que dans ces cas il conviendrait de confier la répression à l'Etat dont les intérêts sont le plus directement touchés, sous réserve du pouvoir disciplinaire də l'Etat d'origine.

12 v. à ce sujet S. Lazareff, op. cit., pp. 187-188.

13 le texte parle d'"élément civil de l'Etat d'origine" - pour une application, v. l'affaire du Commandant Whitley de l'Armée de l'air américaine, qui avait tué un militaire de l'armée de l'air canadienne dans un accident de roulage. La juridiction américaine n'était pas prioritaire, car la victime n'était pas un ressortissant américain et que l'acte n'avait pas été commis dans l'exécution clu service. Pour les décisions françaises (Corr. Corbeil 5 avril 1954, Paris 16 mai 1956 et Cass. ch. cr. 25 mars 1958), v. S. Lazareff, op. cit., pp. 226 et ss. et la note de $M$. Léauté sous le jugement de première instance, Clunet 1954, p. 602. Signalons par ailleurs que nous ne partageons pas l'opinion d'Odile Debbasch (op. cit., p. 133) suivant laquelle l'Etat d'origine aurait une compétence prioritaire du moment que la victime n'a pas la nationalité de l'Etat de séjour. 
gence accomplis dans l'exécution du service" (chiffre 3.a. (ii) ).

l'application de cette règle soulève de nombreuses difficultés, car la notion d'acte accompli dans l'exécution du service n'a pas été précisée. Au cours des réunions préparatoires, plusieurs délégués avaient insisté sur le caractère restrictif de cette notion, en proposant que l'on parle d'acte non seulement accompli dans l'exercice des fonctions, mais aussi dans la limite des fonctions ${ }^{14}$ ou dans l'ordre' des devoirs de l'intéressé. ${ }^{15}$ L'opinion majoritaire fut cependant d'abandonner à l'Etat d'origine le soin de déterminer le contenu de la notion, et plus particulièrement si le délinquant était en service au moment de l'infraction. ${ }^{16}$

Certaines décisions, surtout anciennes, des juridictions des Etats de séjour n'ont pas admis ce droit de l'Etat d'origine, mais ont recherché elles-mêmes le critère du service. Ce' fut notamment le cas en France ${ }^{17}$ et en Turquie. ${ }^{18}$ Des juridictions de fond françaises ont également considéré pouvoir faire application en ce domaine de l'article VIII,

${ }_{14}$ proposition du délégué italien - v. S. Lazareff, op. cit., pp. 195 et ss. 15 proposition du ćélégué canadien - ibid.

16 Telle était notamment la position du délégué américain - ibid.; v. ég. à ce sujet John Gilissen, "L'application des lois pénales...", op. cit., pp. 351-352.

$17 \mathrm{v}$. le jugement de la 17e chambre du Tribunal de première instance de la Seine du 2 juillet 1954 et l'arrêt de la 12e chambre de la Cour d'appel de Paris du 14 décembre 1954, au sujet de l'affaire Sacommandi - v. à ce sujet S. Lazareff, op. cit., pp. 208 et ss.

18 v. l'arrêt cité ci-dessus, note (8) de la 20 ch. cr. de la Cour de cassation et la référence citée. V. ég. Ö. İ. AKİPEK, Nato Kuvvetleri Sözleşmesine Göre Vazife Kavramı ve Türkiye'deki Tatbikatı (Notion de mission d'après la Convention sur les Forces de l'OTAN et la pratique en Turquie), AÜHFM (Revue de la Fac. dr. d'Ankara), vol. XXIV, 1967, nos 1-4, pp. 9-26. Pour d'autres publications en turc sur le même sujet, v. G. ÖKÇÜN, Nato Kuvvetleri Sözleşmesine göre Cezai Kaza Selahiyetinin tesbiti (Etablissement de la compétence juridictionnelle pénale d'après la Convention sur le statut des forces de l'OTAN), Ad. D. (Revue judiciaire), 1960, no: 51, pp. 58-74. V. ég. E. F. Çelik, Nato Kuv. Sözl. göre Kaza Sel. Kullanılması (Utilisation de la compétence juridictionnelle d'après la convention sur le Statut des forces de l'OTAN), IÜHFM (Rev. de la Fac. de Droit d'Istanbul), 1960, XXV, pp. 55-93. 
chiffre 8, de la Convention de Londres, qui autorise le renvoi devant un arbitre d'une éventuelle contestation au sujet du critère du service, mais qui s'applique en réalité uniquement aux problèmes de réparation du dommage..$^{19}$

En Turquie, la question a été réglée par un échange de lettres valant accord sur l'application de l'article' VII, chiffre 3, a, (ii) avec les Etats-Unis. Cet accord, intervenu le 24 septembre 1968, prévoit qu'en cas de contestation sur le critère du service, le Gouvern'ement turc s'engage à s'entretenir de la question avec les autorités militaires de l'Etat d'origine. Si un compromis n'est pas réalisé dans les deux mois, le Gouvernement turc admet le droit prioritaire de l'Etat d'origine, et le Ministre de la justice doit transmettre à la juridiction turque saisie le certificat délivré par le Commandant des forces de l'Etat d'origine' stationnées dans l'Etat de séjour, afin que le dossier soit restitué aux autorités de l'Etat d'origine. ${ }^{20}$

La jurisprudence récente des Etats de séjour admet la déclaration de l'Etat d'origine relative au critère' du service $^{21}$, certaines décisions précisant que la priorité n'est

19 v. les affaires Ensey et Martin, cette dernière ayant donné lieu à l'arrêt de la Ch. cr. de la Cour de cassation du 16 février 1961, qui cassa la décision de fond étendant erronément une disposition de l'article VIII au domaine pénal (v. à ce sujet S. Lazareff, op. cit., pp. 211 et ss.)

20 v. le texte de l'échange de lettres au R.G. du 24 juillet 1969, $\mathrm{n}^{\circ} 13257$ et sur l'ensemble de la question Sahir Erman, "Askeri Ceza Hukuku" (droit pénal militaire), Istanbul, Ed. Sulhi Garan, 1970, pp. 125 et ss. En pratique, les tribunaux turcs de première instance font effectuer une vérification, à l'intervention du Ministère de la justice, par les autorités militaires turques compétentes. Si le quartier général de l'armée turque ne se rallie pas à l'avis des autorités militaires de l'Etat d'origine, la procédure de conciliation est engagée. V. à ce sujet Cass. 2e ch. cr. 3 mars 1970, arrêt $n^{\circ}$ 1255/1780 - affaire D.H. Hilliard; Cass. 1e ch. cr. 29 avril 1972, arrèt $\mathrm{n}^{\circ} 3321$ (décisions non publiées; Archives du Ministère turc de la justice). Nos recherches nous ont permis de constater que beaucoup de décisions des juridictions de première instance étaient cassées pour des motifs de procédure (v. notamment Cass. 2e ch. cr. 26 janvier 1968, arrêt $\mathrm{n}^{\circ}$ 131/432 - affaire Th. Vyse).

21 v. pour la France S. Lazareff, op. cit., et notamment Montpellier, ch. corr. 11 octobre 1961, cité p. 205; pour la Belgique John Gilissen, op. cit., pp. 313 et 351; Liège 19 décembre 1966, R.W. 1966-67, col. 1186. 
subordonnée à aucune autre condition, et notamment pas à la justification de ce que des poursuites auraient été engagées dans l'Etat d'origine. ${ }^{22}$

\section{b) compétence prioritaire de l'Etat de séjour}

Aux termes de l'article VII, chiffre 3, b, "dans le cas de toute autre infraction, les autorités de l'Etat de séjour exercent par priorité leur juridiction".

Ce droit prioritaire est néanmoins dans la pratique limité par le système de la renonciation.

\section{c) renonciation à l'exercice de la compétence prioritaire}

En vertu de l'article VII, chiffre 3, c, l'Etat qui dispose d'une compétence prioritaire peut y renoncer. La décision de renonciation a un caractère définitif. ${ }^{23}$

En ce qui concerne les forces atlantiques stationnées en Allemagne, un Accord complétant la Convention entre les Etats parties au Traité de l'Atlantique Nord a été signé à Bonn le 3 août 1959. Cêt accord prévoit en son article 19 que l'Allemagne renonce d'office à sa priorité en faveur de l'Etat d'origine, à moins qu'en raison de circonstances propres à une affaire déterminée, les autorités allemandes compétentes n'estiment que les intérêts majeurs de l'administration allemande exigent que la juridiction soit exercée par les autorités allemandes. Dans ce dernier cas, elles peuvent révoquer la renonciation dans un délai de vingtet-un jours. ${ }^{24}$

22 v. à ce sujet Cass. fr. ch. cr. 7 mars 1957, Bull. cr. 1958, n 237, p. 419 et RGDIP 1959, p. 1132.

23 v. à ce sujet M. Danse, "Le statut pénal de l'OTAN", op. cit., pp. 338 et SS.; A.D. Belinfante, "Les principes du droit pénal international et les conventions internationales", Ned. tijdschr. v. int. recht 1955 , pp. 244 et ss.; S. Lazareff, op. cit., pp. 225 et ss.; John Gilissen, op. cit., pp. 314 et ss.

24 Signalons que les Etats-Unis ont obtenu un régime de renonciation d'office dans leurs rapports avec la Grèce (accord du 7/9/1956) ainsi que, dans le cadre des conventions bilatérales qui les lient à divers Etats, avec la Corée du Sud, le Groënland, l'Ethiopie, l'Arabie Séoudite, la Libye et les Philippines - v. à ce sujet D. Vignes, "l'affaire Girard", Ann. fr. de dr. int. 1957, p. 311, notes 25 et 26. 
Les cas de révocation sont en pratique assez rares. John Gilissen note qu'il n'y en a eu que quelques-uns au cours dess cinq premières années d'application de l'accord complémentaire..$^{25}$

Les statistiques criminelles allemandes montrent que, de 1967 à 1971, il n'y a eu qu'un nombre extrêmement faible de condamnations prononcées à charge de membres des forces de l'OTAN. ${ }^{26}$

D'une manière générale, il paraît clair que la faculté de renonciation, bien qu'elle soit en principe réservée à chacun des Etats qui bénéficient d'une compétence prioritaire, a surtout été prévue pour permettre à l'Etat d'origine d'exercer une compétence même lorsque la priorité revient à l'Etat de séjour. La Convention précise d'ailleurs que' "les autorités de l'Etat qui a le droit d'exercer par priorité sa juridiction examinent avec bienveillance les demandes de renonciation à ce droit, présentées par les autorités de l'autre Etat, lorsque celles-ci estiment que des considérations particulièrement importantes le justifient", ce qui joue surtout en faveur de l'Etat d'origine. ${ }^{27}$

Comme la renonciation présente un caractère définitif, ${ }^{28}$ il peut se faire que le système de renonciation aboutisse

25 op. cit., p. 316.

26 Les chiffres donnés sont en effet les suivants.

— 1967 : 678 condamnations répressives et 128 administratives (roulage)

- 1968: 787

- 1969: 774 "

- $1970: 744$

- 1971: 966

120

150

$\begin{array}{ll}" & \Rightarrow \\ " & \Rightarrow\end{array}$

27 En ce qui concerne plus particulièrement les forces américaines, René Manhiewicz relève (in "Compétence ratione personae des tribunaux américains et application du statut des forces de l'OTAN aux forces américaines à l'étranger", RIDP 1958, pp. 327-339 et surtout pp. 336-337) que "les autorités militaires sont tenues par leurs ordres d'obtenir dans chaque cas le désistement du juge local." Serge Lazareff (op. cit., p. 288), par une étude des statistiques, arrive à la constatation que les Etats-Unis obtiennent en moyenne des désistements dans $60 \%$ des cas.

28 outre les références citées à la note (21), voir, en ce qui concerne plus particulièrement les forces américaines, J.B. Whitten, "L'exercice de la compétence à l'égard des forces américaines à l'étranger", RGDIP 1959, pp. 17 et ss. 
à l'impunité du délinquant, soit par suite de l'avènement de la prescription. ${ }^{29}$ soit tout simplement parce que l'Etat d'origine, qui tente parfois d'obtenir le dessaisissement plutôt par principe qu'en raison de sa volonté de poursuivre,$^{30}$ n'exerce pas effectivement les poursuites.

S’il est vrai qu'en règle générale, les autorités de l'Etat d'origine ont tendance à se montrer moins indulgentes que celles de l'Etat de séjour, ${ }^{31}$ il nous paraît que cette hypothèse ne peut pas être écartée. Une discrétion très générale est toutefois observée à ce sujet: il manque actuellement de données statistiques sur l'ensemble des forces stationnées dans les pays de l'OTAN. ${ }^{32}$

\section{L'entraide judiciaire secondaire}

L'article VII de la Convention organise une collaboration étroite entre les autorités compétentes de l'Etat d'origine et de l'Etat de séjour, en matière d'arrestation, de remise du délinquant, d'échange de renseignements et de documents, aussi bien pour permettre l'instruction que pour assurer l'information mutuelle au sujet des suites données aux affaires (ceci en cas de juridiction concurrente).

23 V. les exemples cités par R. Manhiewicz, op. cit., pp. 331 et ss, et notamment l'affaire Tarth, relative à un milicien qui avait commis un meurtre en Corée mais dont le crime n'a été découvert que cinq ans après sa démobilisation.

$30 \mathrm{R}$. Manhiewicz note à cet égard que "les autorités militaires et l'opinion publique des Etats-Unis ont une confiance très limitée dans les tribunaux étrangers" (op. cit., p. 336) - v. ég. son commentaire cité à la note (27).

31 En ce qui concerne les forces américaines, on peut relever l'avis de J.B. Whitten, conforme à l'opinion que lui avait exprimée le sénateur Bricker: "En réalité, le militaire américain accusé doit s'estimer plus heureux d'être jugé par un tribunal étranger que de comparaitre devant la Cour martiale américaine; dans les cas de meurtre, par exemple, la peine moyenne infligée aux Américains par la Cour martiale est approximativement de 20 ans de prison, alors que les tribunaux étrangers n'infligent que 6 ans et demi de prison pour le même délit" (op. cit., pp. 14 et 16).

32 Les seules dont on dispose concernent les forces américaines - cf. S. Lazareff, op. cit., p. 287. 
Nous nous permettrons de renvoyer le lecteur sur ces points au texte de la Convention. ${ }^{33}$ Nous souhaitons néanmoins souligner ici que cette entraide joue dans les cas où un Etat bénéficie d'une compétence exclusive, et s'applique donc notamment aux délits politiques. Il en découle que l'Etat de séjour ne peut accorder l'asile politique aux m'embres des forces armées stationnées sur son territoire. On admet par contre que l'asile puisse être accordé par un Etat tiers, même s'il est lui-même partie à l'alliance. ${ }^{34}$

\section{Les effets des jugements}

\section{a) L'effet direct et positif ${ }^{35}$}

L'article VII, chiffre 7, b dispose que "les autorités de l'Etat de séjour examinent avec bienveillance less demandes des autorités de l'Etat d'origine en vue de prêter assistance à celles-ci pour l'exécution des peines d'emprisonnement prononcées sur le territoire de l'Etat de séjour par lesdites autorités conformément aux dispositions du présent article".

Cette disposition n'assure pas la prise en considération de l'effet direct et positif, puisqu'elle ne contient pas d'obligation, mais seulement une recommandation. En outre, elle so limite aux jugements prononcés par l'Etat d'origine dans

33 v. l'article VII, chiffres 5 et 6 ; Nous signalerons cependant que l'entraide secondaire telle qu'elle est prévue par la Convention présente certaines lacunes. C'est ainsi que les autorités canadiennes ont estimé qu'il n'y avait pas lieu de satisfaire à une demande d'audition qui leur avait été adressée par la Turquie portant sur un militaire qui était retourné au Canada à la date de la demande (v. lettre du Ministère turc des affaires étrangères du 30.7.1970 $\mathrm{n}^{\circ}$ 134.632 - Kons./5 - 397b - archives du Ministère de la Justice).

34 v. à ce sujet la réponse de M. Gilisser. suivi son exposé in Droit pénal euror séenns le it. p. 353 - les statistiques relatives au nombre de de $\hat{2}_{c}, a p$. of icains réfugiés à l'étranger confirment cette opinion. Crtèrs ams en effet 8.000 en 1961, 26.000 en 1967, 39.239 en 1968 et on combt vier 1970, environ 700 qui étaient réfugiés à l'étranger, đư Canada (v. ì ce sujet Chronique des faits internationaux, 70, p. 153).

35 Nous entendons par "l'effet direct et positif" l'exécution des peines essentielles et subsidiaires prononcées à l'étranger. 
l'Etat de séjour, et à ceux de ces jugements qui prononcent une peine d'emprisonnement.

Le régime instauré à cet égard n'est donc guère satisfaisant. ${ }^{36}$

\section{b) L'effet direct et négatif 37}

La Convention assure en principe le respect de la règle non bis in idem: "Lorsqu'un inculpé a été jugé conformément aux dispositions de cet article par les autorités d'une partie contractante, et a été acquitté ou, en cas de condamnation, s'il subit ou a subi sa peine ou a été gracié, il ne peut plus être jugé de nouveau sur le même territoire, du chef de la même infraction, par les autorités d'une autre partie contractante" (article VII, chiffre 8, première phrase).

Le respect de cette règle est également assuré par le caractère définitif dé la renonciation.

L'effet direct et négatif ne joue toutefois pas toujours. D'une part, la Convention réserve expressément le droit des autorités de l'Etat d'origine de "juger un membre d'une force pour toute violation des règles de discipline résultant de l'acte ou de l'omission constitutive de l'infraction pour laquelle il a été jugé" (article VII, chiffre 8, deuxième' phrase). Or il paraît évident qu'une infraction tant soit peu grave constituera toujours une faute de discipline. Ensuite, le texte se réfère uniquement à l'interdiction de poursuites sur le même territoire, et ne s'oppose donc pas à ce que l'Etat d'origine prononce un nouveau jugement après İe retour du délinquant dans son pays. Enfin, il exige que le jugement ait été prononcé conformément aux règles de compétence prévues par la Convention. Si par exemple l'Etat d'origine v'nie la priorité de l'Etat de séjour, les juridictions de nuiront refuser d'appliquer la règle

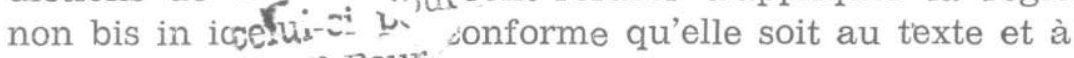

36 v. dans le mêt. ${ }^{33}$ Pour

37 Nous entend fexclusion d'une nouvelle poursuite en raison de la chose we tranger.

$38 \mathrm{~V}$. en ce sems l'arrêt de la Ch. cr. de la Cour de Cassation française du 15 juin 1962, à propos de l'affaire Jensen. Constatant que la 
l'esprit de la Convention, cette conséquence nous parait inconciliable avec les exigences du droit pénal international.

Indépendamment même des cas d'exclusion expressément prévus, on doit en outre rappeler que lorsque le rait peut être considéré commo constitutif d'une infraction distincte, le délinquant sera exposé à de nouvelles poursuites, ce qui arrive notamment en matière de vol d'armes. ${ }^{39}$

\section{E. Conclusion}

Le système de priorité et de renonciation instauré par la Convention de Londres sur le statut des forces de l'OTAN nous paraît dans l'ensemble représenter un modèle appréciable de solution des conflits de juridictions. Nous lui reprochons toutefois d'attribuer à la renonciation de compétence un caractère définitif. Il conviendrait à notre sens au contraire de prévoir, comme cela a été fait dans les conventions européennes régionales de transmission des poursuites, que l'Etat renonçant recouvre sa compétence originaire lorsque les poursuites ne sont pas effectivement exercées dans l'autre Etat.40

Les règles relatives aux effets des jugements nous semblent par ailleurs tout à fait insuffisantes. La Convention n'envisage d'ailleurs même pas les effets indirects, ni les effets directs supplémentaires. ${ }^{41}$

France bénéficiait en l'espèce d'une compétence prioritaire, la Cour a refusé de prendre en considération la condamnation qui avait été prononcée aux Etats-Unis au mépris de cette compétcnce - v. à ce sujet Lazareff, op. cit., p. 259.

$39 \mathrm{v}$. les références citées supra à la note (11).

40 C'est ce qu'avait décidé la Cour d'appel de Paris dans un arrêt du 12 juin 1956, qui fut cassé par un arrêt de la Cour de Cassation du 25 août 1958 (Bull. cr. 1958, n 302, p. 528 et RCDIP 1958, p. 1120). Ie caractère définitif de la renonciation est à notre sens la marque d'une méfiance des juridictions de l'Etat d'origine à l'égard de celles de l'Etat de séjour, méfiance qui n'est pas compatible avec une véritable entraide judiciaire.

41 Les efiets directs supplémentaires consistent, dans le sens où nous entendons ces termes, en la prise en considération du jugement étranger pour l'application de mesures accessoires supplémentaires, telles que les interdictions, déchéances ou incapacités. Quant aux 
Enfin, le sort réservé aux demandes civiles résultant d'un délit ou quasidélit causé par les membres des forces armées stationnées à l'étranger ne nous paraît pas satisfaisant. Bien que l'article VIII de la Convention prévoie certaines facilités pour le recouvrement des dommagesintérêts, le situation des victimes est relativement incertaine: lorsque l'Etat d'origine se charge de l'affaire, elles ne peuvent se constituer partie civile, et sont donc dans l'impossibilité de faire valoir tous les éléments dont elles peuvent disposer; et si l'Etat d'origine classe l'affaire sans suite, il est peu vraisemblable qu'il acceptera d'indemniser la victime, comme l'article VIII l'autorise à le faire, mais sans le lui imposer.

Une protection plus efficace des intérêts de la partie lésée, comme cela a été prévu dans le Traité Benelux sur la transmission des poursuites, nous parait s'imposer, et ce d'autant plus qu'une très grande partie des infractions commises par les membres des troupes stationnées à l'étranger sont dess infractions en rapport avec la circulation automobile..$^{2}$

effets indirects, il s'agit essentiellement de l'imputation de la peine subie à l'étranger.

42 v. à ce sujet R. Meurisse, "Les conséquences pénales et civiles des accidents causés par des véhicules des membres des forces américaines stationnées en France", J.C.P. 1965, doctrine, n ${ }^{\circ} 1899$. 